\title{
CNN-SVO: improving the mapping in semi-direct visual odometry using single-image depth prediction
}

\begin{abstract}
Reliable feature correspondence between frames is a critical step in visual odometry (VO) and visual simultaneous localization and mapping (V-SLAM) algorithms. In comparison with existing VO and V-SLAM algorithms, semi-direct visual odometry (SVO) has two main advantages that lead to state-of-the-art frame rate camera motion estimation: direct pixel correspondence and efficient implementation of probabilistic mapping method. This paper improves the SVO mapping by initializing the mean and the variance of the depth at a feature location according to the depth prediction from a single-image depth prediction network. By significantly reducing the depth uncertainty of the initialized map point (i.e., small variance centred about the depth prediction), the benefits are twofold: reliable feature correspondence between views and fast convergence to the true depth in order to create new map points. We evaluate our method with two outdoor datasets: KITTI dataset and Oxford Robotcar dataset. The experimental results indicate that improved SVO mapping results in increased robustness and camera tracking accuracy. The implementation of this work is available at https://github.com/yan99033/CNN-SVO.
\end{abstract}

